\title{
ERRATA
}

\section{Erratum: Stationary coupled-cluster approaches to molecular properties: A comparative study [Phys. Rev A 54, 250 (1996)]}

\author{
[S1050-2947(97)07402-7] \\ PACS number(s): 31.15.- $\mathrm{p}, 33.20 .-\mathrm{t}, 99.10 .+\mathrm{g}$
}

Nayana Vaval and Sourav Pal

We have found some minor errors in the code for the ECCSD cubic results for $\alpha$ and $\beta$ values reported (in a.u.). The corrected cubic ECCSD $\alpha$ and $\beta$ values obtained are presented below.

The $\alpha_{z z}$ value under the ECCSD cubic column in Table I for $\mathrm{LiH}$ should be 12.89, and for [( $\left.\left.\mathrm{LiH}\right) 2\right] / 2$ should be 12.89. The $\alpha_{z z}$ values under the ECCSD cubic column for Tables II, III, IV, V, and VI should be 8.433, 26.14, 10.579, 4.144, and 13.86, respectively. The $\beta_{z z z}$ value under the ECCSD cubic column in Table I for $\mathrm{LiH}$ should be -745.0 , and for [(LiH)2]/2 should be -745.8. $\beta_{z z z}$ values under the ECCSD column for Tables III and V should be -232.88 and -21.43 , respectively.

Also in Table II, the carbon atom is at the origin. In Tables III and V, SXCCSD results are reported without considering in the functional the terms leading to disconnected diagrams in the equation.

The conclusion, discussion, and other parts of the paper remain unaffected. 